\title{
The Image Sparse Denoising of Redundant Dictionary Based on Filtering Guidance
}

\author{
Yu Xiang ${ }^{1}$ \\ ${ }^{1}$ Department of Information Technology,Tianjin Chengjian University, \\ Tianjin City, China \\ 147940866@qq.com
}

\begin{abstract}
This paper conducts a research on information loss of local feature existing in the image denoising process and puts forward the method of image sparse denoising of redundant dictionary based on filtering guidance. This method utilizes bias noise (additional noise and image errors after denoised image and the corresponding additional noise deviation) for image sparse expression, and extracts the feature information of bias noise to improve the effectiveness of de-noising. In the first place, based on filtering guidance, the method carries out aftertreatment to bias noise still existing after denoise the image. And then, the method, in the basis of this bias noise, designs a new dictionary training method, and obtains redundant dictionary for image processing through self-adaption. Finally, the method extracts featured texture from bias noise image based on the dictionary mentioned above. And it takes advantage of filtering guidance in combination with featured texture extracting information and denoising image to realize image restoration. According to emulated data, the performance of proposed algorithm should be better than the selected comparing algorithm and be equipped with a better visual recovery effect.
\end{abstract}

Keywords: Image de-noising; Redundant dictionary; Guidance filter;Featured texture

\section{Introduction}

Currently, digital imaging technology is widely applied and of great importance, but in the phases of capturing and data transmission, it will be subject to interference from various noises, reducing the quality of transmitting image. Meanwhile, in many specific applications, general requirements involve in high image definition and quality. Therefore, studying how to achieve high-quality denoising and recovery of imager is a very meaningful topic, and it is an urgent need in image application industry. Traditional denoising programs in literatures include frequency domain filtering, median filtering and local filtering, but in the image denoising process, above programs will lead to the partial loss of local images and featured texture data, impacting restoration quality of image. Therefore, this paper mainly studies the generation mechanism of texture loss in popular denoising programs currently. It adopts dictionary training method to have a sparse representation of bias noise, so as to extract featured texture within the scope of image errors. In addition, this paper also designs the Method Noise based Sparse Representation for image denoising algorithm (MNSR). The program, first of all, obtains bias noise of image denoising based on filtering guidance, followed by training the dictionary to achieve obtain redundant dictionary based on bias noise combining with the improved version of K-SVD. Furthermore, the program extracts texture features of image in combination with gained dictionaries and utilizes filtering guidance to restore the images of denoising and extraction results of featured texture, contributing to the achievement of denoising. After adopting bias noise texture features, the peak signal to noise ratio in the program is higher than that before improvement. At the same time, this program can 
effectively maintain the image texture features and details, conductive to improving the visual effect.

\section{Sparse Dictionary Representation}

\subsection{Filtering Guidance}

Supposed that the pending image is $\mathrm{p}$ and guidance image is $\mathrm{I}$, then the outcome is $\mathrm{q}$ after the filtering guidance operation and meets the following conditions:

$$
b_{k}=\bar{p}_{i}-a_{k} u_{k} b_{k}=\bar{p}_{i}-a_{k} u_{k} b_{k}=\bar{p}_{i}-a_{k} u_{k} b_{k}=\bar{p}_{i}-a_{k} u_{k}
$$

In the formula $b_{k}=\bar{p}_{i}-a_{k} u_{k} b_{k}=\bar{p}_{i}-a_{k} u_{k} b_{k}=\bar{p}_{i}-a_{k} u_{k} b_{k}=\bar{p}_{i}-a_{k} u_{k}, \omega_{k}$ represents the position of pixel $k$ relative to the middle position in the $2 \mathrm{D}$ coordinates. $q_{i}$ refers to the first pixel value of output $\mathrm{q} ; p_{i}$ refers to the first pixel value of input $\mathrm{p} ; I_{i}$ refers to the first pixel value of I. $u_{k}$ refers to the mean value of window $\omega_{k}$ 's guidance image below; $\sigma_{k}^{2}$ refers to the variance of window $\omega_{k}$ 's guidance image below; $\bar{p}_{i}$ refers to the mean value of window $\omega_{k}$ 's input image below. They can be summarized in a formula:

$$
\bar{p}_{i}=\frac{1}{|\omega|} \sum_{i \in \omega_{k}} I_{i} p_{i}
$$

\subsection{Dictionary Training}

As for given sample image $\boldsymbol{\beta}=\left(\boldsymbol{\beta}_{1}, \cdots, \boldsymbol{\beta}_{K}\right) \in R^{n \times K}$, the aim of dictionary training is to look up redundant dictionary $\mathbf{D} \in R^{n \times m}$ for the purpose of obtaining sparse representation form of sample, $\boldsymbol{\Gamma}=\left(\boldsymbol{\Gamma}_{1}, \boldsymbol{\Gamma}_{2}, \cdots, \boldsymbol{\Gamma}_{K}\right) \in R^{m \times K}$, so the question is:

$\left\{\begin{array}{ll}\min _{\mathbf{D}, \boldsymbol{\Gamma}} & \|\boldsymbol{\beta}-\mathbf{D} \boldsymbol{\Gamma}\|_{F}^{2} \\ \text { s.t. } & \left\|\boldsymbol{\Gamma}_{k}\right\|_{0} \leq T_{0}\end{array} \quad \forall k\right.$

The dictionary training mentioned above can be solved through MOD and K-SVD method.

\section{Sparse Denosing of Bias Noise}

The proposed method can be divided into three processes: the first one is to denoise image and obtain bias noise based on filtering guidance; the second one is to train redundant dictionary based on bias noise in combination with advanced K-SVD; the third one is to extract image featured texture based on obtained bias noise and use filtering guidance and texture features gained by image denoising for image restoration.

\subsection{Image Bias Noise}

If the additive noise of pending image is additive characteristic noise, its observed features can be described as the form of vector:

$\mathbf{Y}=\mathbf{X}+\mathbf{v}$

In the formula $\mathbf{Y}=\mathbf{X}+\mathbf{v}$, parameter $\mathbf{v}$ refers to white noise, whose mean value is 0 and variance is $\sigma^{2}$. Y is the pending image polluted by additive noise; $\mathrm{X}$ is the pending image. Denoise image based on filtering guidance to gain image $\mathrm{X}$ and its corresponding bias noise $\Delta$ and its form can be described as:

$\mathbf{Y}=\hat{\mathbf{X}}+\Delta$

Bias noise $\Delta$ gained through filtering guidance include texture feature $\mathrm{H}$ of the image. In the process of filtering guidance denoising image, image featured texture will be loss. 


\subsection{Redundant Dictionary Training}

Bias noise $\Delta$ gained here obtains redundant dictionary $\mathbf{D} \in R^{n \times m}$ through learning. Then it divides the bias noise $\Delta$ into $\mathrm{K}$ blocks. Image block will overlap and the size of it is $\sqrt{n} \times \sqrt{n}$. Image block can be described as $\Delta_{k} \in R^{n}$. Randomly select image blocks to train redundant dictionary $\mathrm{D}$, representing $P=\left\{p_{1}, p_{2} \cdots, p_{L}\right\}$. So the issue mentioned above can be described as:

$$
\begin{cases}\min _{\mathbf{D}, \mathbf{Q}} & \|\mathbf{P}-\mathbf{D Q Q}\|_{F}^{2} \\ \text { s.t. } & \left\|\mathbf{q}_{l}\right\|_{0} \leq T_{0} \quad \forall l\end{cases}
$$

In the (4) formula $\begin{cases}\min _{\mathbf{D}, \mathbf{Q}} & \|\mathbf{P}-\mathbf{D Q}\|_{F}^{2} \\ \text { s.t. } & \left\|\mathbf{q}_{\boldsymbol{l}}\right\|_{0} \leq T_{0} \quad \forall l \quad \mathbf{Q}=\left\{\mathbf{q}_{1}, \mathbf{q}_{2} \cdots \mathbf{q}_{L}\right\} \in R^{m \times L} \quad \text { is a sparse matrix. The }\end{cases}$ optimized solution procedure of (4) is as follows:

\section{Step 1: Sparse Coding}

As for dictionary D, the solution updates its sparse matrix Q. So the optimized solution can be represented by sequence sparse coding form:

$\forall l\left\{\begin{array}{l}\min _{\mathbf{q}_{l}}\left\|\mathbf{p}_{l}-\mathbf{D} \mathbf{q}_{l}\right\|_{2}^{2} \\ \text { s.t. }\left\|\mathbf{q}_{l}\right\|_{0} \leq T_{0}\end{array}\right.$

The Formula (5) is a traditional sparse coding form and the adopting method K-SVD is based on OMP program to find a solution. According to [16 17] literatures, $L_{0}$ norm has a better effect for image sparse expression. Hereto, here we try to find a minimum solution to Formula (5) based on $L_{0}$ norm.

If properly selecting parameter $u$, the Formula (6) can be transformed into:

$\min _{\mathbf{q}_{l}}\left\{-\mu\left\|\mathbf{q}_{l}\right\|_{0}+\left\|\mathbf{D} \mathbf{q}_{l}-\mathbf{p}_{l}\right\|_{2}^{2}\right\}$

Based on Gaussian ${ }^{L_{0}}$ norm, the Formula (6) can be deduced:

$n-\lim _{\delta \rightarrow 0} \sum_{i=1}^{n} \exp \left(-\beta_{i}^{2} / 2 \delta^{2}\right)=\left\|\mathbf{x}_{k}\right\|_{0}$

In this formula, $\beta_{i}$ refers to the element $i$ of vector $\mathbf{q}_{l}$, so according to

$F_{\delta}\left(\mathbf{q}_{l}\right)=-\sum_{i=1}^{n} \exp \left(-\beta_{i}^{2} / 2 \delta^{2}\right)$

It can be concluded that under the $\delta \rightarrow 0$, the issue (6) is equivalent to:

$\min _{\mathbf{q}_{l}} L_{\delta, u}\left(\mathbf{q}_{l}\right)=-u F_{\delta}\left(\mathbf{q}_{l}\right)+\left\|\mathbf{D} \mathbf{q}_{l}-\mathbf{p}_{l}\right\|_{2}^{2}$

In this formula, $u$ is the relevant sparse parameter and its iteration form can be deduced based on steepest descent method:

$\mathbf{q}_{l}=\mathbf{q}_{l}-t \nabla L_{\delta, u}\left(\mathbf{q}_{l}\right)$

In the Formula (9), $t$ refers to step size, representing gradient in image $\boldsymbol{q}_{l}$ :

$\nabla L_{\delta, u}\left(\mathbf{q}_{l}\right)=\frac{u}{\delta^{2}} \boldsymbol{\Lambda} \mathbf{q}_{l}+2 \mathbf{D}^{\mathrm{T}}\left(\mathbf{D} \mathbf{q}_{l}-\mathbf{p}_{l}\right)$

In this formula, diagonal matrix is:

$\boldsymbol{\Lambda}=\operatorname{diag}\left\{\exp \left(-\beta_{1}^{2} / 2 \delta^{2}\right), \exp \left(-\beta_{2}^{2} / 2 \delta^{2}\right), \cdots, \exp \left(-\beta_{n}^{2} / 2 \delta^{2}\right)\right\}$ 
Select step size $t$ and make it meet the condition:

$L_{\delta, u}\left(\mathbf{q}_{l}-t \nabla L_{\delta, u}\left(\mathbf{q}_{l}\right)\right)<L_{\delta, u}\left(\mathbf{q}_{l}\right)$

Step 2: update of dictionary training

Fix the sparse matrix $\mathbf{Q}$ and update the dictionary $\mathbf{D}$, so the dictionary updating process can be described as:

$\min _{\mathbf{D}}\|\mathbf{P}-\mathbf{D Q}\|_{F}^{2}$

Supposed that $\mathbf{q}_{T}^{j}$ is the element in $j$ of sparse matrix $\mathbf{Q}$. If the form of dictionary is $\mathbf{D}=\left(\mathbf{d}_{1}, \mathbf{d}_{2}, \cdots, \mathbf{d}_{m}\right)$, it can be concluded that $\mathbf{Q}=\left(\mathbf{q}_{T}^{1}, \mathbf{q}_{T}^{2}, \cdots, \mathbf{q}_{T}^{m}\right)^{T}$. Then the further conclusion is:

$$
\begin{aligned}
& \|\mathbf{P}-\mathbf{D Q}\|_{F}^{2}=\left\|\mathbf{P}-\sum_{j=1}^{m} \mathbf{d}_{j} \mathbf{q}_{T}^{j}\right\|_{F}^{2}=\left\|\left(\mathbf{P}-\sum_{j \neq j_{0}} \mathbf{d}_{j} \mathbf{q}_{T}^{j}\right)-\mathbf{d}_{j_{0}} \mathbf{q}_{T}^{j_{0}}\right\|_{F}^{2} \\
& \text { In combination with } \mathbf{E}_{j_{0}}=\mathbf{P}-\sum_{j \neq j_{0}} \mathbf{d}_{j} \mathbf{q}_{T}^{j}, \text { the process of dictionary updating is: }
\end{aligned}
$$

$\min _{\mathbf{D}}\left\|\mathbf{E}_{j_{0}}-\mathbf{d}_{j_{0}} \mathbf{q}_{T}^{j_{0}}\right\|_{F}^{2}$

According to the Formula (14), it can be achieved to update atoms in dictionary based on SVD method and synchronously update its corresponding sparse coefficient, specific details refer to literature [2]. Based on the above process, dictionary $\mathrm{D}$ can be accessed.

\subsection{Image Denosing}

The image texture feature $\mathrm{H}$ extracted from self-adaption bias noise based on dictionary $\mathrm{D}$ and utilizes filtering guidance to obtain image $\mathrm{X}$ and texture feature $\mathrm{H}$ after denoising and then restores image.

Extract texture features and obtain dictionary D sparse expressions in bias noise of image block $\Delta_{k} \in R^{n}$. The formula is a sparse code and its form is:

$$
\forall k\left\{\begin{array}{l}
\min _{\boldsymbol{a}_{k}}\left\|\boldsymbol{\Delta}_{k}-\mathbf{D} \boldsymbol{\alpha}_{k}\right\|_{2}^{2} \\
\text { s.t. }\left\|\boldsymbol{\alpha}_{k}\right\|_{0} \leq T_{0}
\end{array}\right.
$$

The step obtains sparse expression $\boldsymbol{\alpha}_{k}$ of image block based on the minimized solution by $L_{0}$ norm described in the quarter 2.2, so the texture feature of image flock in bias noise is $\hat{\mathbf{H}}_{k}=\mathbf{D} \boldsymbol{\alpha}_{k}$. Assemble the image blocks and average filtering the overlapped areas, then texture features form can be deduced:

$$
\hat{\mathbf{H}}=\left(\sum_{k} \mathbf{R}_{k}^{T} \mathbf{R}_{k}\right)^{-1}\left(\sum_{k} \mathbf{R}_{k}^{T} \hat{\mathbf{H}}_{k}\right)
$$

In the Formula (16), $\mathbf{R}_{k}$ is mainly used for extracting corresponding matrix of image blocks in group $k$. So the obtained texture feature is $\hat{\mathbf{H}}$ and denoised image gained from filtering guidance, then it can be concluded that:

$\mathbf{X}^{*}=\hat{\mathbf{X}}+\hat{\mathbf{H}}$

Texture features mentioned above keep the algorithm's characteristics: firstly, it can enhance the characteristics of two groups of elements bounded by dictionary and its dictionary-based optimal update form is better than advanced K-SVD and other updated 
forms; secondly, sparse coding process is coupled in dictionary update process of the two groups ; thirdly, dictionary training process is able to solve the non-convex problem only by iteration for limited times without precisely calculating the minimum point; finally, each time dictionary updates, there only involves a dictionary. And the dictionary is fixed, which can stop the pollution from other forms of structures, thus realizing the effective division of two groups of elements.

\section{Algorithm Description}

\subsection{Procedures of Algorithm}

The method of image denoising of redundant dictionary based on extracted filtering guidance is specifically processed in the algorithm 1 .

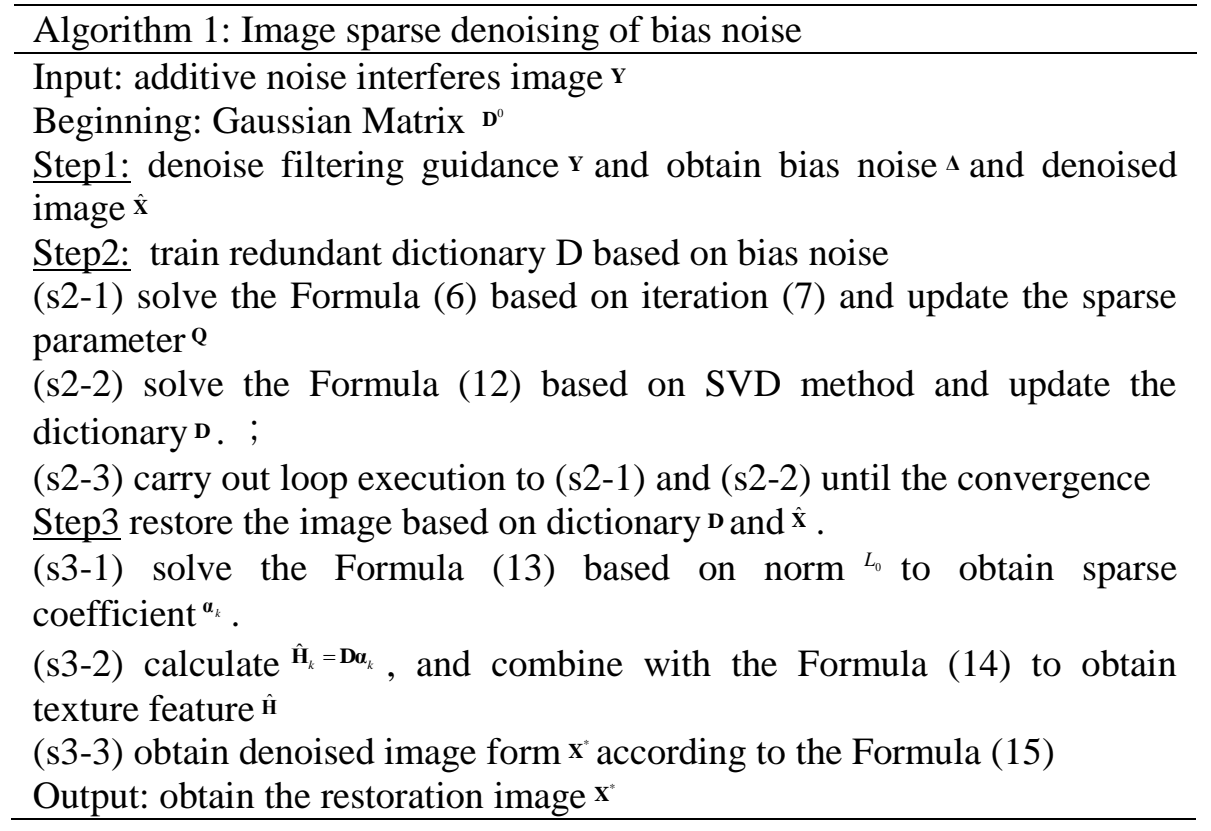

Annotation:

(1) guidance decision: denoise the noised image $\mathbf{Y}$ by using filtering guidance;

(2) select parameter $u$ : the optimization process of the Formula (13) is regarded as the process of optimization with several purposes

\subsection{Convergence Analysis}

It mainly aims at analyzing the convergence of the iteration process of the Formula (7).

Theorem 1: the sequence that the iteration of Formula (7) optimizes the solution to problems tends to be convergent.

Demonstration: according to:

$$
\begin{aligned}
& \nabla L_{\delta, u}\left(\mathbf{q}_{l}\right)^{\mathrm{T}} \nabla L_{\delta, u}\left(\mathbf{q}_{l}\right)>0 \\
& \quad \text { and supposed } \mathbf{d}_{k}=\nabla L_{\delta, u}\left(\mathbf{q}_{l}\right) \text {, then it can be concluded: } \\
& -\nabla L_{\delta, \lambda_{k}}\left(\tilde{\mathbf{x}}_{k}\right)^{\mathrm{T}} \mathbf{d}_{k}<0
\end{aligned}
$$


and the gradient of ${ }^{\mathbf{d}}$ is descending. So it is equipped with step ${ }^{t}$ and meets the conditions:

$$
L_{\delta, u}\left(\mathbf{q}_{l}-t H \nabla L_{\delta, u}\left(\mathbf{q}_{l}\right)\right)<L_{\delta, u}\left(\mathbf{q}_{l}\right)
$$

Thereby, the Formula (16) obtained iterative sequence is declined. And as the function $L_{\delta, u}\left(\mathbf{q}_{l}\right)$ has differentiable continuous features, if setting the initial value, the obtained iterative sequence is bounded. Overall, data series obtained from the iteration of the Formula (7) is convergent. Q.E.D.

\section{Experimental Analysis}

Contrast algorithm selects K-SVD algorithm [1]. Algorithm experiments can be divided into the following four groups: Experiment 1 contrasts different noises of mean variance to gain PSNR value resulting noise change situation; Experiment 2 carries on contrast from the perspective of vision; Experiment 3 contrasts the difference of algorithm performance under the condition of overlapped pixels; Experiment 4 contrasts algorithm performance differences when the number of atoms is not the same.

Parameter setting: set the size of image block bias noise equivalent to $5 \times 5$ and overlapping pixels to 4 . Randomly select 20,000 groups of images to train dictionary. Set the number of atoms in the dictionary $\mathbf{D}$ to 150 , then $\mathbf{D} \in R^{25 \times 150}$ can be concluded. Hardware setting: as for CPU, select quad-core $3.0 \mathrm{GHz}$, for RAM, select $8 \mathrm{~Gb}$ mainframe and for algorithm evaluation index, select peak signal to noise ratio (PSNR).

Experiment 1: maintenance of texture features' effects

Firstly, select three legends--boat, barbara and house to have a comparison test. Table 1 , deduces PSNR value under different mean variance noise. According to data in Table 1 , under the same setting, and the results gained by MNSP image restoration program in this paper is superior to comparative programs.

Table 1. Comparison of PSNR Values of Three Kinds of Denoising Algorithms

\begin{tabular}{ccccccc}
\hline \multirow{2}{*}{$\sigma$} & \multicolumn{2}{c}{ barbara } & \multicolumn{2}{c}{ boat } & \multicolumn{2}{c}{ house } \\
& K-SVD & MNSR & K-SVD & MNSR & K-SVD & MNSR \\
\hline 20 & 30.78 & 31.69 & 30.35 & 31.15 & 32.76 & 33.35 \\
25 & 29.37 & 30.51 & 29.25 & 30.34 & 32.24 & 32.79 \\
30 & 28.47 & 29.68 & 28.54 & 29.48 & 31.15 & 31.56 \\
50 & 25.36 & 26.43 & 25.89 & 26.65 & 27.89 & 28.38 \\
100 & 21.87 & 23.26 & 22.79 & 23.47 & 24.46 & 25.86 \\
\hline
\end{tabular}

Secondly, choose a remote sensing image in a place of Changping District from 2005 to 2010 .

Through texture feature extraction and spectral feature extraction forms, people can vary region extraction. The original image is shown in Figure 1a, and Figure $1 b$, and the extracted image is showed in Figure 1c, and 1d, respectively. 


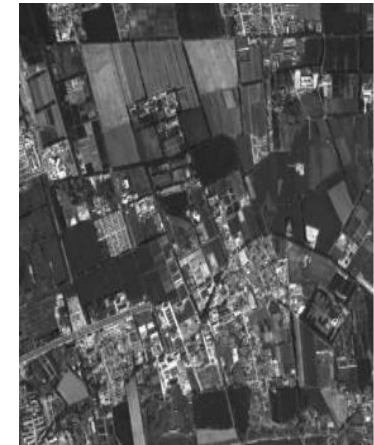

(a) in 2005

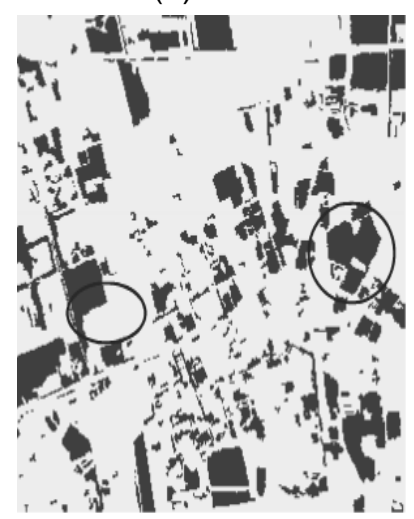

(a) Changes in Extracting Texture Features

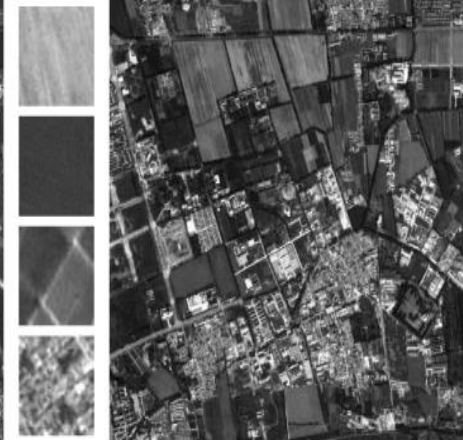

(b) in 2010

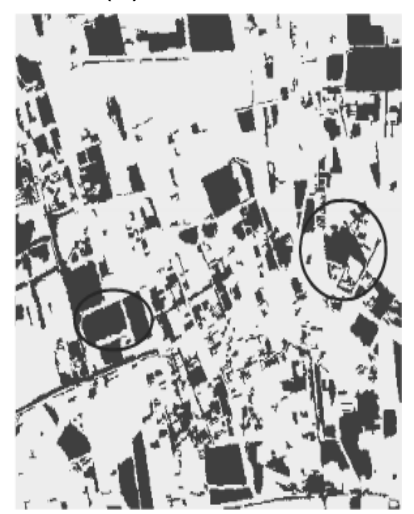

(b) Changes in Extracting Spectrum Features

Figure 1. Texture Features' Effects

Figure 1, shows the contrast between texture feature extraction and spectrum feature extraction in a remote sensing image of a place in Changping District from 2005 to 2010. Its aim is to see the extraction results in change areas (such as the marked circle area in Figure 1c, and 1d). From the comparison results, the extraction results in change areas based on texture feature extraction is better than that of spectrum feature extraction, as the latter causes feature interference for different seasonal drops, thus there exists obvious wrong extraction phenomenon.

This experiment is based on the visual denoising performance of comparison algorithm in three groups of remote sensing images. Select mean variance indicator as $\sigma=30$. Figure 1 , shows the visual denoising performance of three groups of remote sensing images. According to the images in Figure 2, shows that the effect of image reconstruction of MNSR program designed in this paper is more detailed and more approximate to the original image in visual sense.

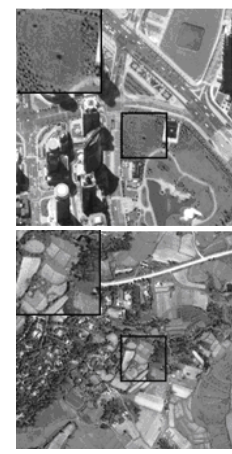

(a)

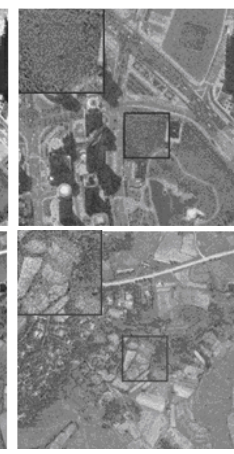

(b) 


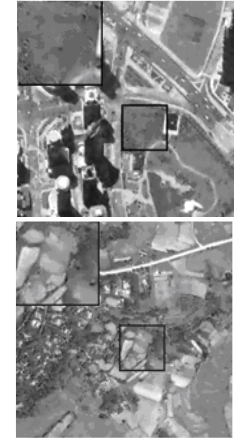

(c)

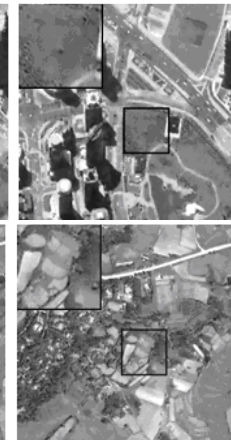

(d)

Figure 2. Denoising Algorithm Visual Effect [(a) Image; (b) Image Noise; (c) K-SVD Algorithm; (d) MNSR Algorithm]

Experiment 2: contrast the influence of pixel overlapping quantity

This experiment shows the image representing the influence of overlapping pixels quantity on denoising effect of algorithm image. Set the parameter $\sigma=30$. Figure 2, shows the comparison condition between MNSP program when the number of overlapping pixels and PSNR indicator of K-SVD program. This PSNR indicator is the mean value of three pieces of remote sensing images in Experiment 1. According to Figure 3, as the number of overlapping pixels is increasing, the indicators in MNSR program and K-SVD program are improved and MNSR program is always superior to K-SVD program.

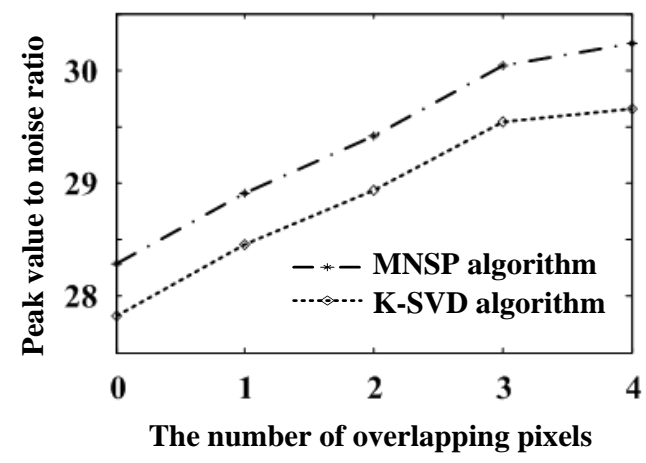

Figure 3. The Influence of the Number of Pixels

Experiment 3: contrast the influence of atoms in dictionary

The comparison test lists atom quantity's influence on the effect of denosing algorithm method. Set the parameter $\sigma=50$ and suppose the remaining stimulation settings do not change simulation, the variation range of atom quantity is from 100 to 1000 . What listed in Figure 4, is the PNSR indicator comparison condition gained from dictionaries with different auto quantity. Similar to Experiment 2, it also selects PSNR indicators' mean value in three groups of images to carry on comparison. According to the data comparison in Figure 3, when the number of atoms in dictionary is not less than 150, PSNR indicators in MNSR program have reached saturation. 


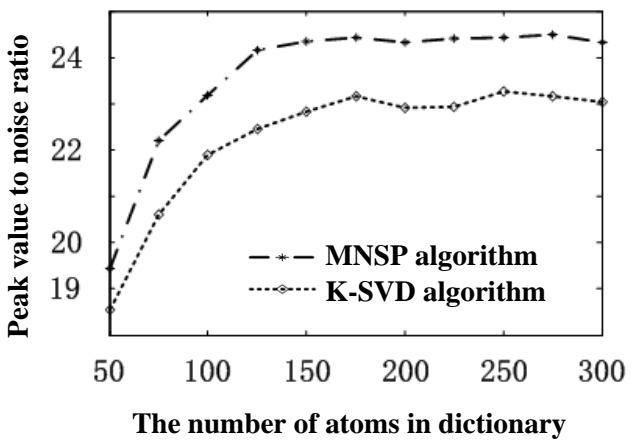

Figure 4. The Influence of the Number of Atoms in a Dictionary

\section{Conclusion}

In order to solve the problem that it is difficult to maintain image details after denoising and inadequate protection on texture features in existing de-noising programs, this paper puts forward image sparse denoising program of redundant dictionary based on filtering guidance. Firstly, this program denoises image and obtains bias noise based on filtering guidance. Then it extracts texture features included in image noise based on dictionary training and sparse matrix representation, thus achieving the denoising quality improvement in details. According to comparative tests, the program proposed here has a more proper peak signal-to-noise ratio than comparing algorithm, and can provide better protection for image texture features and detail features, so as to improve the effect of visual denoising.

\section{Acknowledgment}

The research is supported by Tianjin Xinghai plan(KJXH2014-13).

\section{References}

[1] Y. Geng, J. Chen, R. Fu, G. Bao and K. Pahlavan, "Enlighten Wearable Physiological Monitoring systems", On-Body RF Characteristics Based Human Motion Classification Using a Support Vector Machine, vol. 99, (2015), pp. 1-16.

[2] X. Song and Y. Geng, "Distributed Community Detection Optimization Algorithm for Complex Networks", Journal of Networks, vol. 9, no. 10, (2014), pp. 2758-2765.

[3] K. Pahlavan, P. Krishnamurthy and Y. Geng, "Localization Challenges for the Emergence of the Smart World", Access, IEEE, vol. 3, no. 1, (2015), pp. 1-11.

[4] J. He, Y. Geng, Y. Wan, S. Li and K. Pahlavan, "A cyber physical test-bed for virtualization of RF access environment for body sensor network", Sensors Journal, IEEE, vol. 13, no. 10, (2013), pp. 38263836.

[5] Z. Lv, A. Tek and F. D. Silva, "Game on, science-how video game technology may help biologists tackle visualization challenges", PloS one, vol. 8, no. 3, (2013), pp. 57990.

[6] T. Su, W. Wang and Z. Lv, "Rapid Delaunay triangulation for randomly distributed point cloud data using adaptive Hilbert curve", Computers \& Graphics, vol. 54, (2016), pp. 65-74.

[7] D. Jiang, Z. Xu and Z. Lv, "A multicast delivery approach with minimum energy consumption for wireless multi-hop networks", Telecommunication Systems, (2015), pp. 1-12.

[8] C. Fu, P. Zhang and J. Jiang, "A Bayesian approach for sleep and wake classification based on dynamic time warping method", Multimedia Tools and Applications, (2015), pp. 1-20.

[9] J. Hu and Z. Gao, "Modules identification in gene positive networks of hepatocellular carcinoma using Pearson agglomerative method and Pearson cohesion coupling modularity", Journal of Applied Mathematics, vol. 2012, (2012).

[10] Z. Lv, "Wearable smartphone: Wearable hybrid framework for hand and foot gesture interaction on smartphone", Computer Vision Workshops (ICCVW), (2013) IEEE International Conference on, IEEE, (2013), pp. 436-443.

[11] Y. Lin, J. Yang and Z. Lv, "A Self-Assessment Stereo Capture Model Applicable to the Internet of Things", Sensors, vol. 15, no. 8, (2015), pp. 20925-20944. 
[12] J. Yang, S. He and Y. Lin, "Multimedia cloud transmission and storage system based on internet of things", Multimedia Tools and Applications, (2015), pp. 1-16.

\section{Author}

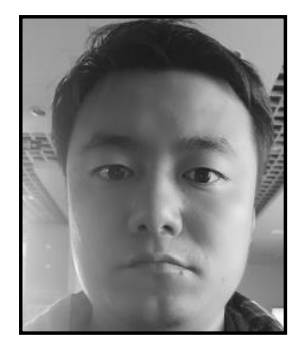

Yu Xiang, received her M.S. Degree in internet engineering from Estonian Business, School of Information Technology and Telecommunications in Tallinn, Estonia. He is currently a lecturer in Tianjin Cheng Jian University. His research interest is mainly in the area of Computer Software, Internet and Electrical Integration. He has published several research papers in scholarly journals in the above research areas and has participated in several books. 